\title{
Indoxil sulfato induce senescencia y liberación de microvesículas en células endoteliales que promueven la calcificación vascular.
}

\author{
Beatriz Escobar ${ }^{1,}$ a, Guillermo Bodega ${ }^{2}$, Julia Carracedo ${ }^{3,4}$, Rafael Ramírez ${ }^{1}$, Matilde Alique ${ }^{1}$ \\ 1. Departamento de Biología de Sistemas, Facultad de Medicina y Ciencias de la Salud, Universidad de Alcalá. Alcalá \\ de Henares, Madrid, Spain. 2. Departamento de Biomedicina y Biotecnología, Facultad de Ciencias, Universidad de \\ Alcalá. Alcalá de Henares, Madrid, Spain. 3. Departamento de Fisiología Animal (II), Facultad de Biología, Universidad \\ Complutense de Madrid, Madrid, Spain. 4. Institute of Investigation, Hospital 12 de Octubre, Madrid, Spain.
}

a. beatriz.ed98@gmail.com

V Congreso de Señalización Celular, SECUAH 2020.

16-18 de marzo, 2020. Universidad de Alcalá. Alcalá de Henares, Madrid. España.

Palabras clave: senescencia; microvesículas; calcificación vascular; células endoteliales; toxinas urémicas

\section{Resumen}

Antecedentes: Tanto los ancianos como los pacientes con enfermedad renal crónica (ERC) tienen una mayor probabilidad de sufrir enfermedades inflamatorias cardiovasculares (EICVs), las cuales pueden asociarse a la calcificación vascular como inicio de las mismas. Las células endoteliales tienen una senescencia prematura como mecanismo fisiopatológico en la ECV asociada a ERC. Hemos demostrado que las microvesículas (MVs) producidas por estas células senescentes, debido a su capacidad de comunicación intercelular, pueden iniciar dicha calcificación. Además, las toxinas urémicas, como el indoxil sulfato (IS), que se relacionan con la ERC, también pueden generar una senescencia endotelial precoz, lo que puede secretar MVs que inicien la calcificación vascular. Todo esto permite que las MVs puedan ser útiles para su caracterización como biomarcadores tempranos y/o diana terapéutica en estas patologías.

Materiales y métodos: Se trataron células endoteliales (HUVEC) con IS. La senescencia se cuantificó por el método de $\beta$-galactosidasa. Por citometría de flujo se aislaron y se caracterizaron las MVs producidas por las HUVEC senescentes, y se utilizaron para el tratamiento de células musculares lisas (HASMC) durante 9, 20 y 30 días. En HASMC se valoraron los depósitos de calcio por el colorante rojo alizarina. A los 30 días, mediante el kit de fenolsufoneftaleina, se cuantificó el contenido de calcio. Mediante PCR cuantitativa se determinó en HASMC la expresión de genes pro-calcificantes y por western blot la expresión de un marcador específico de HASMC (SM22 $\alpha)$.

Resultados: El tratamiento con IS indujo senescencia precoz en HUVEC (control: $7,07 \pm 2,87 \%$ vs. IS: $72,90 \pm 13,09 \%, p<0.0001$ ) y se observó un incremento en la producción de MVs (control: $1,68 \pm 1.00$ vs. IS: $9.92 \pm 4,29 \mathrm{MVs} / \mathrm{célula}, \mathrm{p}<0.005)$. Las MVs procedentes de células con senescencia precoz por el efecto del IS indujeron calcificación en HASMC, en un proceso asociado a desregulación en la expresión de genes procalcificantes (Runx2, BMP2). Además, las MVs generadas en HUVEC tratadas con IS producen un proceso de desdiferenciación de las HASMC (expresión de SM22 $\alpha$ ).

Conclusión: Las células endoteliales que tienen una senescencia precoz como consecuencia del estrés oxidativo generado por las toxinas urémicas son capaces de producir MVs que promuevan la calcificación vascular. Su cuantificación en plasma permite que se pueda utilizar como biomarcador para valorar la progresión de la enfermedad en sujetos susceptibles y para diseñar dianas terapéuticas que eviten la generación o la presencia en el espacio extracelular de MVs procalcificantes.

Cita: Escobar, Beatriz; Bodega, Guillermo; Carracedo, Julia; Ramírez, Rafael; Alique, Matilde (2020) Indoxil sulfato induce senescencia y liberación de microvesículas en células endoteliales que promueven la calcificación vascular. Actas del V Congreso de Señalización Celular, SECUAH 2020. 16-18 de marzo, 2020. Universidad de Alcalá. Alcalá de Henares, Madrid. España. dianas 9 (1): e202003fa05. ISSN 1886-8746 (electronic) journal.dianas.e202003fa05 http://www3.uah.es/dianas?e202003fa05.

URI http://hdl.handle.net/10017/15181

Copyright: ( Escobar B, Bodega G, Carracedo J, Ramírez R, Alique M. Algunos derechos reservados. Este es un artículo open-access distribuido bajo los términos de una licencia de Creative Commons ReconocimientoNoComercial-SinObraDerivada 4.0 Internacional. http://creativecommons.org/licenses/by-nc-nd/4.0/

\section{INTRODUCCIÓN}

Los pacientes con enfermedad renal crónica (ERC) se caracterizan por sufrir una disfunción endotelial producida por la acumulación de toxinas urémicas, entre otros desencadenantes. Al igual que ocurre en otras situaciones, en la ERC se sugiere que esta disfunción endotelial es el elemento inicial en la cascada de eventos que va a conducir al desarrollo de enfermedad cardiovascular (ECV), principal causa de morbilidad y mortalidad entre los enfermos con ERC. Conocer cómo se produce la cascada de daño que se inicia en el endotelio es un objetivo clave para prevenir la progresión de las ECV [1]. 
Junto con la disfunción endotelial, el desarrollo de calcificaciones vasculares (CV) también es frecuentemente observado en los enfermos con ERC [2] y en las personas mayores [3]. De hecho, la determinación de calcificación es utilizada como un elemento predictor de morbilidad y mortalidad cardiovascular en estos pacientes, así como de evolución clínica y pronóstico [4]. El diagnóstico de la misma se realiza por análisis radiológicos y no hay ningún biomarcador específico descubierto [5].

Estudios recientes sugieren que la producción de vesículas extracelulares (VE) por las células endoteliales es un elemento fisiológico clave, puesto que vehiculan señales epigenéticas a través de las cuales la célula endotelial mantiene la homeostasis vascular. Pero también hay numerosos estudios clínicos y experimentales que demuestran que cuando las VE endoteliales vehiculan señales alteradas promueven daño en otras células vasculares $\mathrm{y}$, en consecuencia, actúan como un mecanismo fisiopatológico promoviendo la progresión del daño vascular que llevará al desarrollo de ECV [6].

Estas VE son estructuras en forma de esfera delimitadas por membrana que se producen por la mayor parte de las células en respuesta a un estímulo fisiológico y/o patológico para posteriormente ser liberadas al medio extracelular. Se clasifican en 3 subtipos, donde las microvesículas (MV), también conocidas como micropartículas, serían las más relevantes en nuestro estudio [7]. Las MV influyen sobre la regulación de la homeostasis al participar en la comunicación intercelular. Sin embargo, en condiciones patológicas, las células dañadas son capaces de liberar distintas cantidades de MV que se caracterizan por transportar moléculas diferentes, promoviendo una serie de cambios que pueden desencadenar daños en otras células de tejidos adyacentes [8-10]. En concreto, aquellas producidas por células endoteliales se pueden cuantificar en sangre periférica y usarse como posible biomarcador para evaluar el daño endotelial $[3,11,12]$. De este modo, su caracterización fenotípica podría proporcionar información sobre el mecanismo por el cual las células endoteliales causan lesiones vasculares y, por tanto, median el desarrollo de las enfermedades cardiovasculares (ECV) [13,14].

La calcificación en las células vasculares va a producirse por una cadena de señales que terminan en una transformación osteogénica y que comienza con las MV liberadas por las células [15] al actuar como una señal en el proceso de calcificación donde se regula el equilibrio entre el fósforo inorgánico y el calcio [3]. Además, las células musculares lisas vasculares (CMLV) se vuelven susceptibles a la acción de las MV y sufren una desdiferenciación progresiva en su fenotipo, haciendo que se asemejen a los osteoblastos [16].

La temprana identificación de la calcificación vascular en pacientes con ERC o incluso en etapas previas a este proceso, junto con el entendimiento del mecanismo fisiopatológico de la $\mathrm{CV}$, podría ser esencial para prevenir la progresión de la ECV. El problema hasta la fecha es la falta de conocimiento sobre los mecanismos celulares y moleculares involucrados en el desarrollo de CV. Diferentes estudios han identificado a las VE, producidas por células inflamatorias o las propias células musculares lisas, como un elemento de enucleación a partir de la cual se precipita el proceso de calcificación en las células musculares lisas [17]. Sin embargo, estos estudios experimentales no dan respuesta a una pregunta clave, cómo elementos circulantes en sangre periférica como las toxinas urémicas, hacen que se dañen células como las musculares lisas que, por su situación anatómica, no están en contacto directo con las mismas.

En este estudio hemos planteado como hipótesis el hecho de que toxinas urémicas y otros elementos circulantes en sangre periférica inducen senescencia y daño endotelial. Estas células senescentes producirán VE alteradas que vehiculan señales anómalas y promueven daño en otras estructuras vasculares, como las células musculares lisas, promoviendo, en consecuencia, la calcificación vascular y, con ella, las ECV. Este enfoque podría ser útil como herramienta de diagnóstico en futuros estudios para identificar pacientes con riesgo de sufrir $\mathrm{CV}$ y, asimismo, desarrollar nuevas terapias dirigidas a la mejora de la salud.

\section{Materiales y métodos}

\section{Cultivos celulares}

Se cultivaron células endoteliales de vena umbilical humana (HUVEC, CC-2517, lote 323352; Lonza) en medio EGM (Lonza) suplementado con suero bovino fetal (FBS; Sigma) inactivado por calor al $10 \%$. Los cultivos se mantuvieron a $37{ }^{\circ} \mathrm{C}$ en condiciones de $5 \%$ de $\mathrm{CO}_{2}$ y $95 \%$ de humedad en el medio EGM, (EBM, CC-3121; CC-4133, Lonza) que contiene extracto de cerebro bovino, ácido ascórbico, hidrocortisona, factor de crecimiento epidérmico y gentamicina / anfotericina-B. Los experimentos con HUVEC se realizaron en células con $<9$ pases $(\mathrm{PD}<20$ ). La tasa de duplicación de la población (PD)se calculó utilizando la fórmula $\mathrm{PD}=[\ln \{$ número de células cosechadas $-\ln$ \{número de células sembradas $\}$ ) / $\ln 2]$.

Se obtuvieron células de músculo liso aórtico humano (HASMC, CC-2571, Lonza) y se mantuvieron en el medio SmGM-2 (Lonza; CC-3181) suplementado con $20 \%$ de FBS (Sigma) a $37{ }^{\circ} \mathrm{C}$ en una atmósfera humidificada con 5\% de $\mathrm{CO}_{2}$. Se usaron los pases 5-7 para los experimentos. 


\section{Diseño experimental en HUVEC tratadas con IS}

Las HUVEC se sembraron el día anterior (día 0) al tratamiento en medio EGM (Lonza) suplementado con $10 \%$ de FBS (Sigma). Al día siguiente (día 1), las células endoteliales se trataron durante 3 días con 250 $\mu \mathrm{M}$ de IS (indoxil sulfato; Sigma) disuelto en DMSO (Sigma). En el día 4 se cambiaron los medios para HUVEC y, al día siguiente (día 5), se recogieron sobrenadantes de las células (Figura 1). En cada experimento se agruparon los sobrenadantes según las condiciones y las MV se aislaron como se describe en el siguiente apartado.

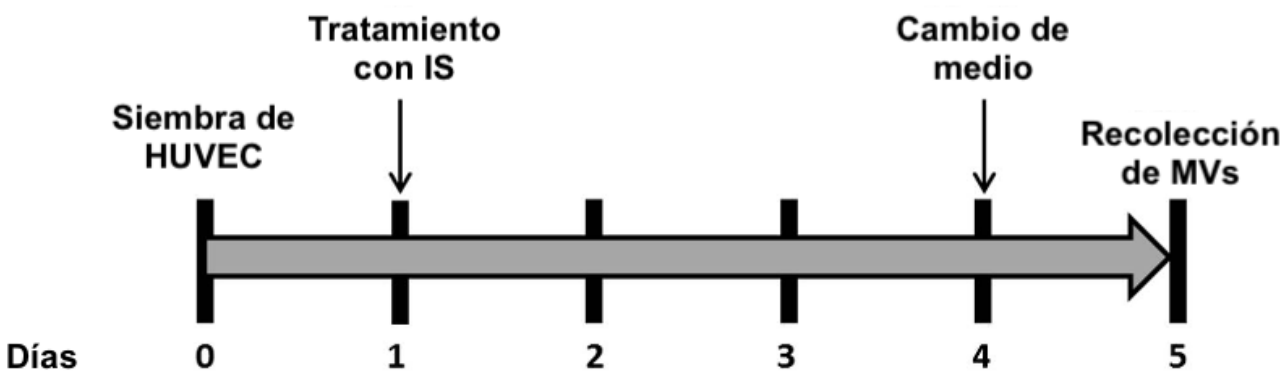

FIGURA 1. Diagrama esquemático del diseño experimental para obtener un modelo de senescencia en células endoteliales (HUVEC) tratadas con IS.

\section{Aislamiento y cuantificación de MV}

Los sobrenadantes del cultivo celular se centrifugaron en serie (15 minutos a 3.000 rpm para eliminar células y restos celulares, y 30 minutos a $14.000 \mathrm{rpm}$ para concentrar las MV) en centrifugadoras Eppendorf, según la Sociedad Internacional de Trombosis y Hemostasia $\left[{ }^{18}\right]$.

Las MV agrupadas de los cultivos de HUVEC tratados con IS y control se caracterizaron siguiendo las pautas de la Sociedad Internacional de Vesículas Extracelulares (ISEV) $\left.{ }^{19}\right]$. Las MV agrupadas de las células endoteliales control y tratadas con IS se mezclaron en cantidades iguales y luego se caracterizaron y cuantificaron según tamaño usando el citómetro de flujo Beckman Coulter Cytomic FC 500 con el software CXP.

\section{Determinación de la actividad de la $\beta$-galactosidasa}

Las HUVEC control y las tratadas con IS se tiñeron para evaluar la actividad de $\beta$-galactosidasa (SA- $\beta$ gal) mediante un kit de senescencia (Abcam, Catálogo \# ab65351) de acuerdo con el protocolo del fabricante. Para ello, el día 5 del diseño experimental, las células endoteliales control y las tratadas con IS se lavaron con PBS y se fijaron en solución de fijación durante 12 minutos a temperatura ambiente. A continuación, se lavaron en PBS para eliminar la solución de fijación y se incubaron con SA- $\beta$-gal a 37 ${ }^{\circ} \mathrm{C}$ durante $16 \mathrm{~h}$ sin $\mathrm{CO}_{2}$. Al final del período de incubación, las células $\mathrm{SA}-\beta$-gal-positivas se identificaron como células teñidas de azul bajo un microscopio óptico. El número de células positivas se contó y normalizó con el número de células totales en el mismo campo. El porcentaje de células positivas para SA- $\beta$-gal se calculó haciendo el recuento de 8 campos microscópicos seleccionados al azar (aumento $100 \mathrm{X})$.

\section{Tratamiento de HASMC con MV y determinación de niveles de mineralización}

Las HASMC se sembraron en placas de 24 pocillos a una densidad de 10000 células/pocillo, y se cultivaron con $50000 \mathrm{MV} / \mathrm{mL}$ durante 9, 20 y 30 días en medio DMEM suplementado con FBS al 2\%. Las células expuestas a DMEM suplementado con FBS al 2\% con fósforo inorgánico 2,0 mM (Pi; condición procalcificante) se usaron como control positivo.

La detección y cuantificación de depósitos minerales en HASMC se realizó mediante rojo de alizarina, (método de tinción cualitativa). Las muestras se fijaron (50\% de etanol 5 mins y 95\% de etanol 5 mins), se tiñeron durante 5 mins con rojo de alizarina $\mathrm{S}(40 \mathrm{mM}, \mathrm{pH} 4,2$; Sigma) y se enjuagaron con $50 \%$ de etanol. Al final del período de incubación se utilizó un microscopio de luz invertida con contraste de fase y se tomaron imágenes de las células (Optika; aumento 100X).

\section{Cuantificación de depósitos de calcio}

Una vez finalizado el período de incubación máximo de 30 días, se cuantificaron los niveles de calcio. Las células musculares lisas se descalcificaron con $\mathrm{HCl} 0,6 \mathrm{~N}$ durante toda la noche a $4{ }^{\circ} \mathrm{C}$. El contenido de calcio de los sobrenadantes se determinó por espectrofotometría, usando un kit que contiene colorante de fenolsulfoneftaleína (no. DICA-500, kit de ensayo de calcio QuantiChrom; BioAssay Systems). 
Posteriormente, las células se lavaron tres veces con PBS (Sigma) y se solubilizaron en $\mathrm{NaOH} 0,1 \mathrm{M} / \mathrm{SDS}$ al 1\%. El contenido de proteínas procedentes de las células musculares lisas se midió usando el kit de ensayo de proteína BCA (Pierce), y la cantidad total de calcio se normalizó con respecto al contenido de proteína total.

\section{PCR en tiempo real}

El ARN total se extrajo de las HASMC tratadas con MV, utilizando el kit de purificación de proteínas nativas y ARN mirVana PARIS (Ambion), siguiendo las instrucciones del fabricante. El ADNc se sintetizó mediante el kit de ADNc de alta capacidad (Applied Biosystems, Foster City, California, EE. UU.), utilizando $2 \mu \mathrm{g}$ de ARN total según las instrucciones del fabricante. Las reacciones en cadena de la polimerasa en tiempo real (PCR) se realizaron utilizando el sistema de detección de secuencia ABI Prism 7500 (TaqMan ${ }^{\circledR}$ Universal Master Mix II, No AmpErase ${ }^{\circledR}$ UNG; Applied Biosystems). Para la identificación de los genes que participan en la calcificación de las células musculares lisas se utilizaron las sondas Taqman: Runx2 (Hs001047973_m1) y BMP2 (Hs00154192_m1), y HPRT1 (Hs02800695_m1), donde este último fue utilizado para normalizar. Las copias de ARNm se calcularon utilizando un valor de umbral comparativo $(\mathrm{Ct})$. La transformación relativa se determinó usando métodos de $2^{\Delta \Delta \mathrm{Ct}}$, usando de control HASMC tratadas con MV; y sus niveles se normalizaron a los niveles de expresión de HPRT1.

\section{Western blot}

Los extractos del control y las células endoteliales tratadas con IS, así como las HASMC control y tratadas con MV se lisaron usando el tampón de lisis de extracción de proteínas CytoBuster (Millipore), al que se le añadieron inhibidores de proteasa y fosfatasa (Roche). El contenido de proteína total de los lisados se cuantificó utilizando el kit de determinación de proteínas BCA (Pierce), utilizando BSA como estándar en la curva. A continuación, se cargaron en los geles de proteínas entre 20-50 $\mu \mathrm{g}$ de proteína/pocillo y se separaron mediante geles de SDS/PAGE en condiciones reductoras. Las muestras se transfirieron a membranas de nitrocelulosa (BioRad), se bloquearon en TBS que contenía Tween 20 al $0,1 \%$ de SDS y leche desnatada en polvo al 5\% durante 1 hora a temperatura ambiente. Se utilizó el anticuerpo primario SM22 $\alpha$ (Santa Cruz Biotechnology, sc-53932, dilución 1/500, 22 kDa) y $\alpha$-tubulina (T5158; Sigma) como control de carga. Después del lavado, las membranas se incubaron con anticuerpos secundarios Novex (1: 5000). Las bandas se visualizaron con el sustrato Luminata Crescendo Western HRP (Millipore). La eficacia de la transferencia de proteínas se evaluó mediante tinción con Red Ponceau (Sigma). Las bandas se cuantificaron usando el software Image J (NIH) y los niveles de proteína se normalizaron con los niveles anti- $\alpha$-tubulina en HASMC.

\section{Análisis estadístico}

Los datos se expresaron como: medias \pm SD. La prueba $t$ de Student o la prueba de Mann-Whitney se usaron para comparar variables que estaban distribuidas normalmente o no, respectivamente, en dos grupos. De lo contrario, la significación estadística se determinó utilizando ANOVA, seguido de la prueba de Kruskal-Wallis. Se consideró que un valor de $\mathrm{p}<0,05$ era estadísticamente significativo, en el que $* \mathrm{p}<0,05, * * \mathrm{p}<0,01 \mathrm{y} * * * \mathrm{p}<0,001$. Para todo ello se utilizó el programa GraphPad Prism 6.

\section{Resultados}

\section{El tratamiento con IS induce senescencia en HUVEC}

El tratamiento con IS en HUVEC produjo un aumento en el número de células senescentes al observar un incremento significativo en comparación con las células control, lo cual se averiguó al teñir las células con $\beta$-galactosidasa (SA- $\beta$-gal). Junto a esto se observó una diferencia en la morfología de estas células senescentes, adquiriendo una morfología celular aplanada y agrandada típica (Figura 2A).

Al calcular el porcentaje de senescencia se observó que éste era diverso, a pesar de que todos los experimentos de exposición a IS se realizaron en las mismas condiciones. Como consecuencia de esta observación y en base a estudios previos realizados por nuestro grupo $\left[{ }^{20}\right]$, se establecieron dos grupos con diferentes porcentajes de senescencia con el tratamiento de IS. Un grupo tenía un porcentaje intermedio de células senescentes $(42,85 \pm 4,72 \%)$, mientras que el otro tenía un porcentaje elevado de células senescentes $(72,90 \pm 13,09 \%)$, en comparación con el porcentaje de células endoteliales no tratadas $(7,07 \pm 2,87 \%)$ (Figura $2 \mathrm{~B})$. 
A
Control

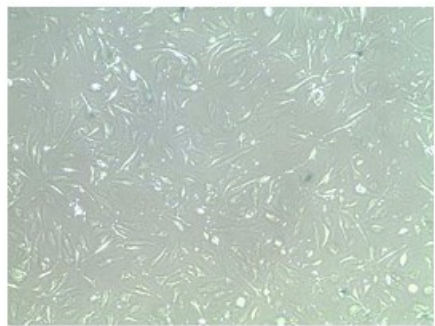

Senescencia intermedia inducida por IS
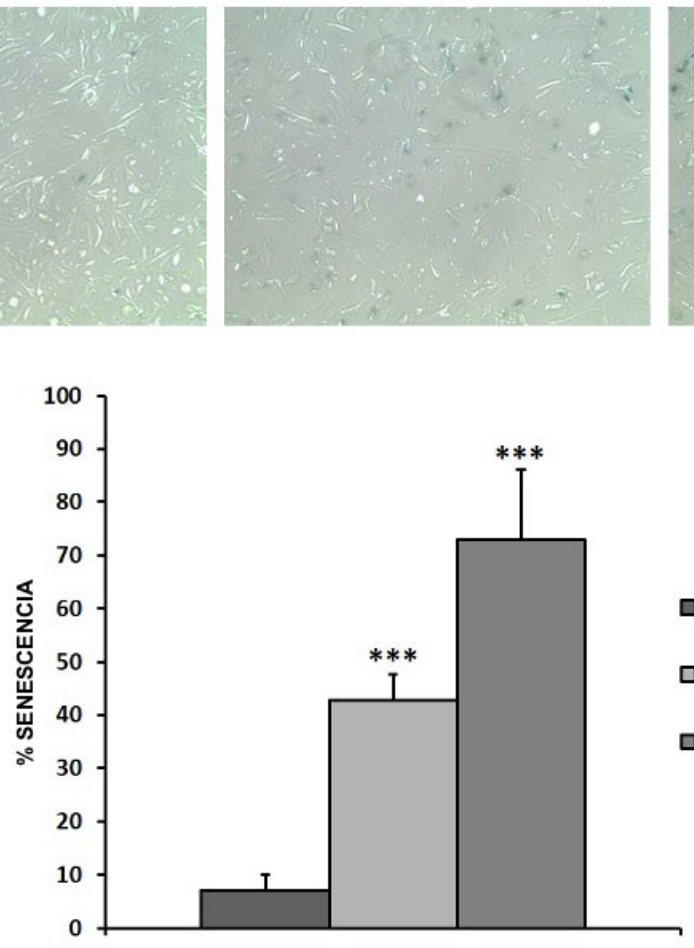

Senescencia elevada inducida por IS

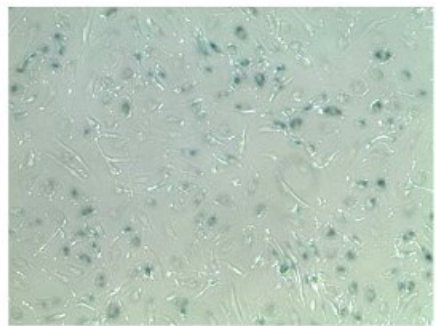

B

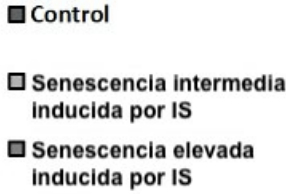

FIGURA 2. Senescencia en HUVEC. Las HUVEC desarrollan un fenotipo senescente después del tratamiento con IS $(250 \mu \mathrm{M}$ durante 3 días). El porcentaje de HUVEC senescentes se determinó mediante tinción de $\beta$-galactosidasa. (A) Imágenes de microscopía de contraste de fase. (B) Los datos representan medias $\pm \mathrm{SD}$ y se expresan como porcentaje de células totales. Control $(\mathrm{n}=6)$; Senescencia intermedia inducida por IS $(n=4)$; Senescencia elevada inducida por IS $(n=4)$; aumento, $\mathrm{x} 100 . * * * \mathrm{p}<0,001$. Control de HUVEC vs. HUVEC tratadas con IS.

\section{Liberación de MV por IS}

El número de MV en HUVEC controles y tratadas con IS fue determinado para ver si se podía observar una mayor producción de MV cuanto mayor era el grado de senescencia. El número de MV se evaluó mediante citometría de flujo (número/ $\mu \mathrm{L}$ ), y el recuento total de células endoteliales se cuantificó con la cámara de Neubauer. La relación se expresó como el cociente entre el número de MV/número de HUVEC (ratio MV/célula). Cabe destacar el aumento que se observó en la producción de MV por célula durante la senescencia elevada inducida por IS $(9,92 \pm 4,29, \mathrm{n}=4)$, en comparación con el control $(1,68 \pm 1,00, \mathrm{n}=$ $6)$. También se observó un aumento, pero no significativo, $(2,88 \pm 1,17, \mathrm{n}=4)$ en el grupo de senescencia intermedia inducida por IS (Figura 3).

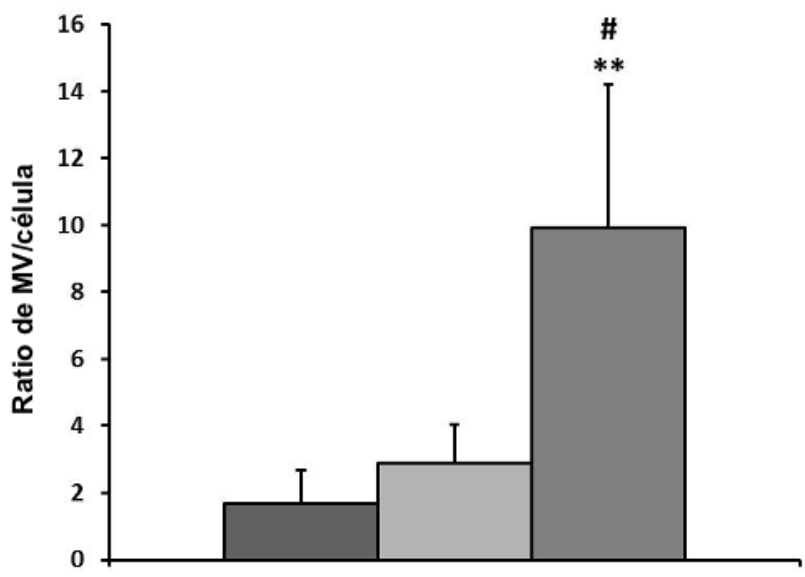

$\square$ Control

$\square$ Senescencia intermedia inducida por IS

$\square$ Senescencia elevada inducida por IS

FIGURA 3. Cociente de MV por célula generadas por el tratamiento con IS en HUVEC. La relación se expresa como el cociente entre el número de MV y el número de células HUVEC en cada experimento (relación de MV por célula). Control $(\mathrm{n}=6)$; Senescencia intermedia inducida por IS $(\mathrm{n}$ $=4)$; Senescencia elevada inducida por IS $(n=4) .{ }^{* *} \mathrm{p}<0,01$, Control de HUVEC vs. senescencia elevada inducida por IS. \# $\mathrm{p}<0,001$, senescencia intermedia inducida por IS vs. senescencia elevada inducida por IS. 


\section{Las MV liberadas de HUVEC tratadas con IS inducen calcificación en HASMC}

El posible efecto procalcificante de las MV generadas por HUVEC tratadas con IS se evaluó al tratar HASMC con MV (50000 MV/mL) durante 9, 20 y 30 días. Los depósitos de calcio se evaluaron en los diferentes días por medio del colorante rojo de alizarina en HASMC (Figura 4).

Ambos grupos de MV procedentes de células con diferentes grados de senescencia indujeron calcificación después de 30 días de tratamiento, especialmente aquellas MV procedentes de células endoteliales que alcanzaron una senescencia elevada con el tratamiento con IS. A su vez, las células HASMC tratadas con MV control no generaron ningún depósito de calcio. Como control positivo de calcificación se incubaron las HASMC con fosfato, ya que es procalcificante.

La confirmación de estos hallazgos se sustentó con la cuantificación del contenido de calcio que se llevó a cabo mediante un método colorimétrico basado en el reactivo de fenolsulfoneftaleína a los 30 días de tratamiento con MV (Figura 4B). En el día 30 hubo un aumento significativo de los depósitos de calcio en HASMC causado por el efecto de las MV de HUVEC tratadas con IS. Además, se observaron diferencias significativas entre el desarrollo de los depósitos de calcio entre los diferentes grupos de MV generadas por el tratamiento del IS en las HUVEC (Figura 4B).

A

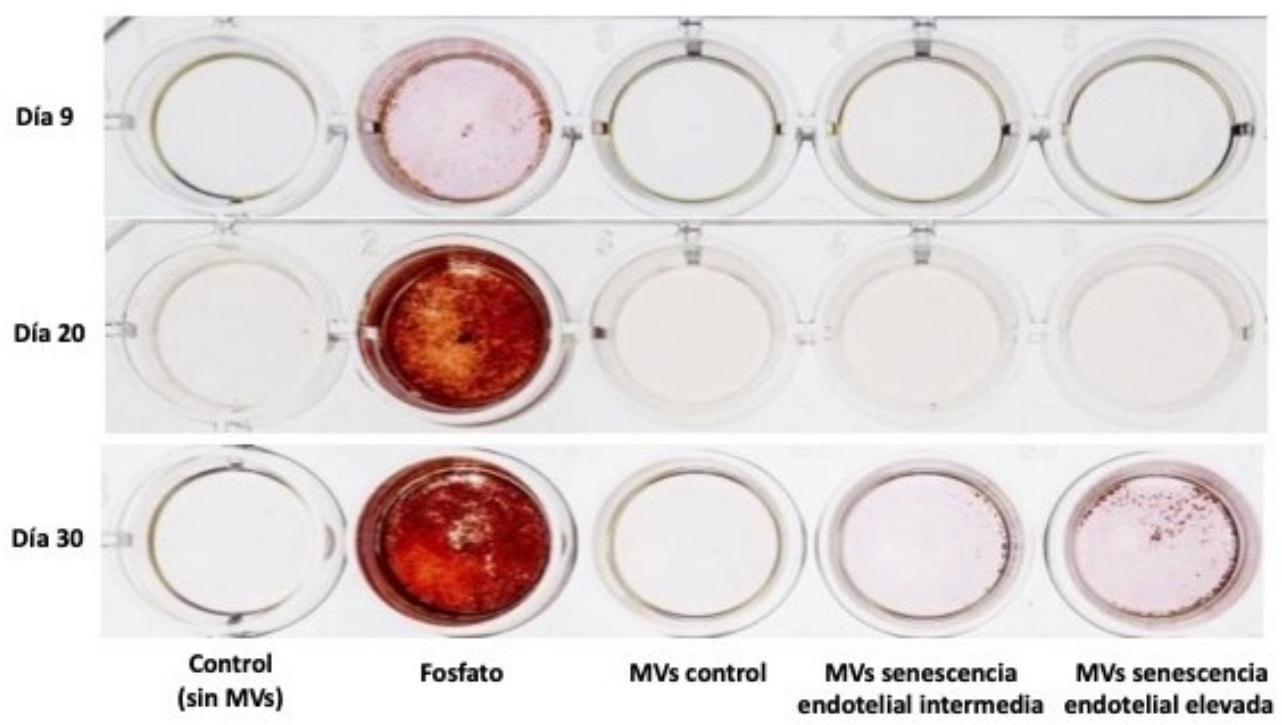

B

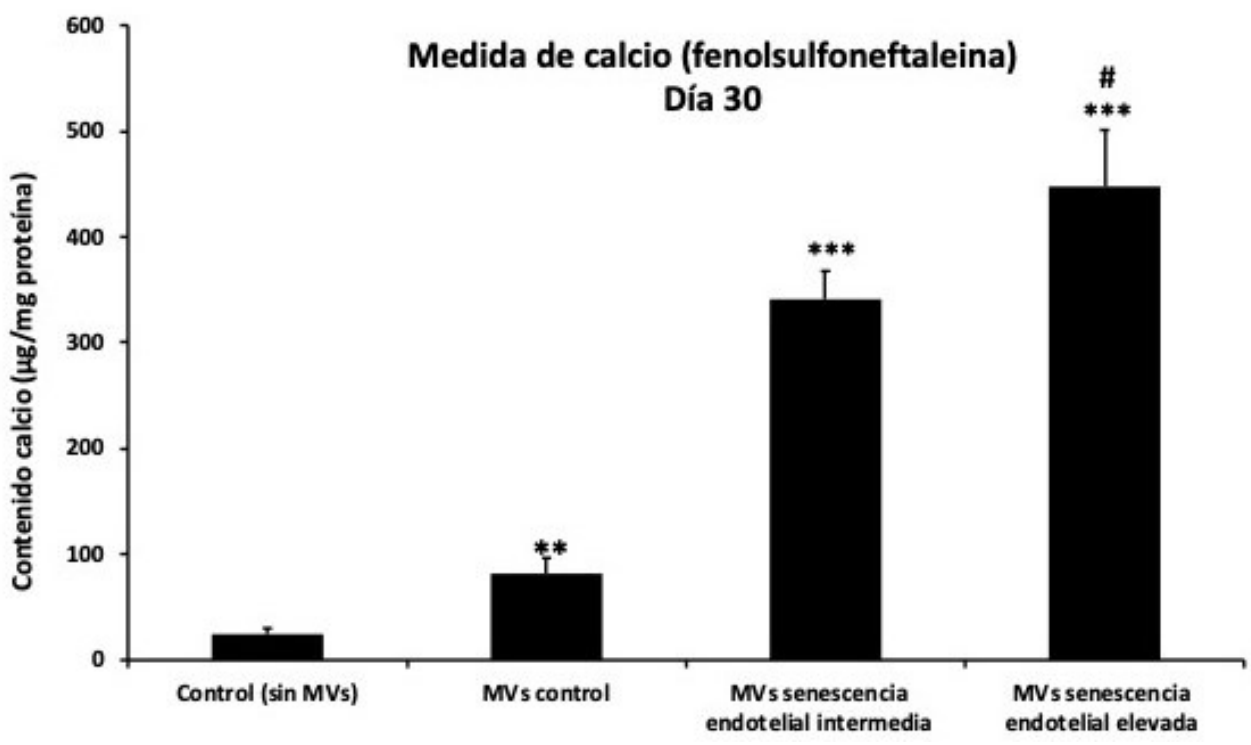

FIGURA 4. Inducción de calcificación en HASMC. Se cultivaron HASMC con $50000 \mathrm{MV} / \mathrm{mL}$ del control y HUVEC tratadas con IS durante diferentes días de tratamiento. (A) La calcificación cualitativa se evaluó mediante la tinción de HASMC con rojo de alizarina. (B) El contenido de calcio se determinó por espectrofotometría usando fenolsulfoneftaleína después de 30 días de tratamiento con MV en HASMC. Los histogramas representan el contenido de calcio de las células, expresado como $\mu \mathrm{g} / \mathrm{mg}$ de proteína total. Los datos representan las medias $\pm \mathrm{SD}$ de 3 experimentos independientes. $^{* *} \mathrm{p}<0,05$ vs. control sin MV; ${ }^{* * *} \mathrm{p}<0,001$ vs. control $\sin \mathrm{MV}, \mathrm{y} \# \mathrm{p}<0,001$ vs. MV de senescencia intermedia inducida por IS al mismo tiempo. 


\section{Las MV de HUVEC tratadas con IS incrementan la expresión de genes procalcificantes en HASMC}

El aumento de la expresión de genes inductores de calcificación en HASMC fue analizado mediante qPCR. Los resultados demostraron una inducción significativa de la expresión de los genes procalcificantes Runx2 y BMP2 (Figura 5A). Esto se pudo observar en las HASMC que habían sido tratadas con MV procedentes de células endoteliales con un grado de senescencia tanto intermedio como elevado. Concretamente, las MV de células senescentes intermedias aumentaron los niveles del gen Runx2 con el tratamiento con MV a los 30 días y, por otro lado, la expresión del gen BMP2 aumentó tanto con 20 como con 30 días de tratamiento con MV. Sin embargo, para las HASMC tratadas con MV obtenidas de células con senescencia elevada, los niveles de Runx2 se incrementaron tras 9, 20 y 30 días y los niveles de BMP2 aumentaron tras 20 y 30 días de tratamiento.

Este efecto inductor de calcificación se confirmó mediante el cambio fenotípico de las HASMC, y se evalúo la expresión de un marcador de HASMC específico (SM22 $\alpha$ ). Sus niveles se evaluaron mediante Western blot y se observó una disminución de la expresión proteica de SM22 $\alpha$ en HASMC tras 20 y 30 días de tratamiento con MV de HUVEC tratadas con IS (Figura 5B).

A
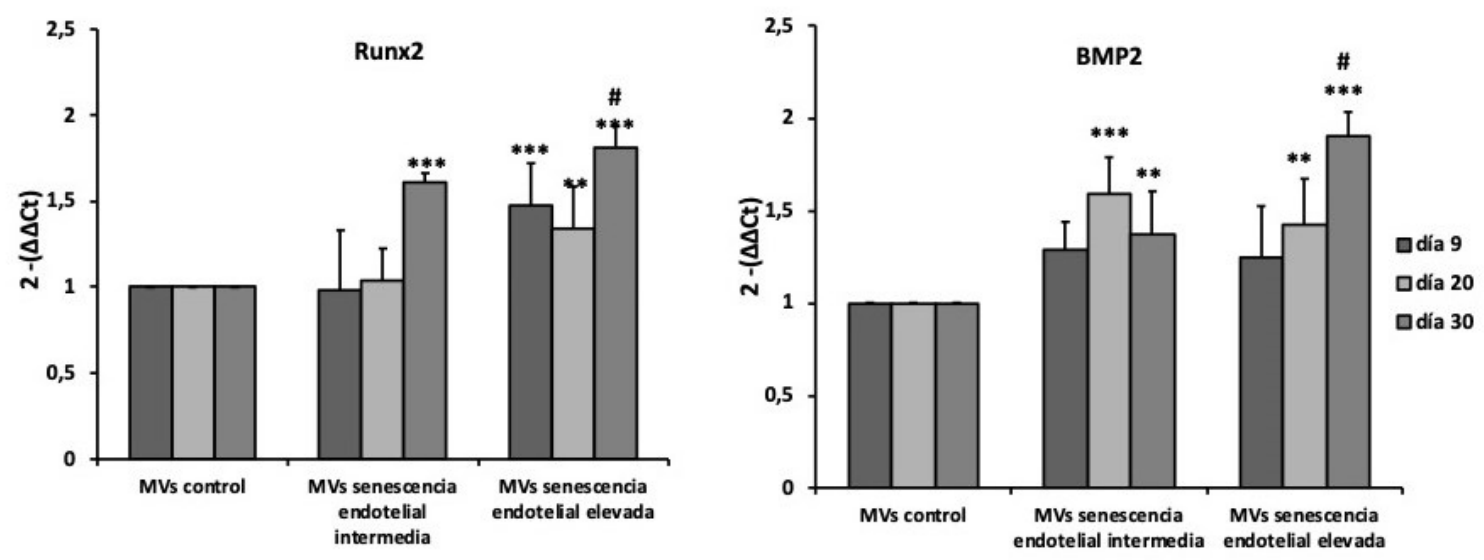

B

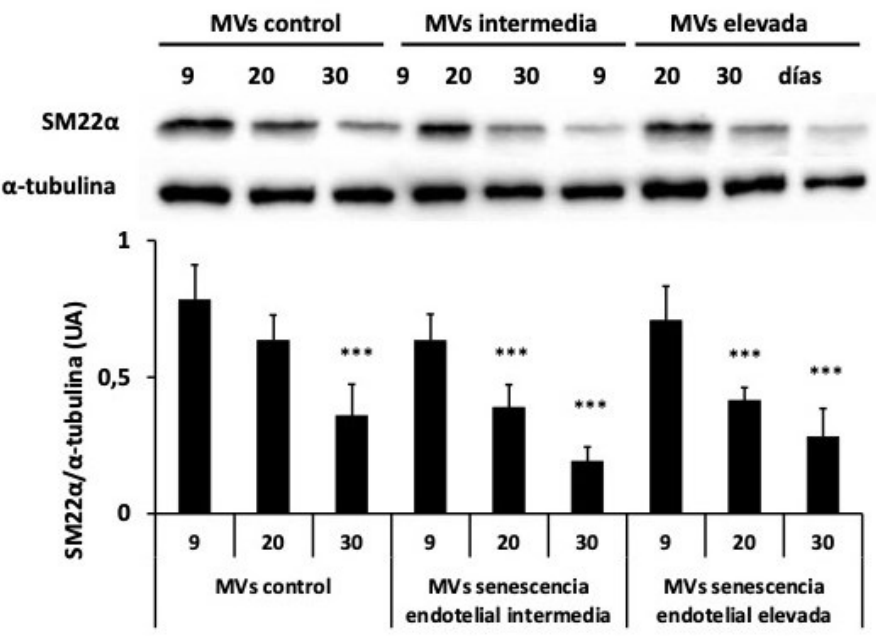

FIGURA 5. Marcadores de calcificación de HASMC. (A) Análisis qPCR de ARNm de Runx2 y BMP2 obtenidos de HASMC tratadas con MV usando el método $\triangle$ Ct. Se usó ARNm de HPRT1 para la normalización. $\mathrm{n}=2$ por duplicado. ${ }^{* *} \mathrm{p}<0,05$ vs. MV control; $* * * \mathrm{p}<0,001$ vs. MV control; \#p $<0,05$ vs. MV de senescencia intermedia inducida por IS al mismo tiempo. (B) Análisis de Western blot de SM22 $\alpha$ de HASMC tratadas con MV durante diferentes días. El sondeo con $\alpha$-tubulina permite confirmar la carga igual de proteínas. El gráfico presenta datos sobre el análisis de bandas densitométricas normalizadas con $\alpha$-tubulina en términos de unidades arbitrarias (UA). $n=2$ por duplicado. $* * * \mathrm{p}<0,001$ vs. 9 días al mismo tiempo.

\section{Discusión}

En la ERC se observa una alta propensión a CV, que se asocia al daño inducido en las células vasculares, ya sea por las toxinas urémicas o por otras sustancias [5]. Identificar precozmente esta calcificación podría ser un proceso crítico en la prevención de la progresión de la ECV como principal causa de morbimortalidad en estos pacientes. El problema es que hoy en día no existe un método de diagnóstico para evaluar las etapas iniciales en la formación de $\mathrm{CV}$ o los pacientes con ERC que tienen riesgo de 
desarrollarla, haciendo que se limiten las posibilidades en la mejora de la enfermedad, puesto que esta patología se detecta cuando el proceso ya es irreversible [5].

Recientemente, diferentes estudios han descrito a las MV como promotoras de la CV [ $\left.{ }^{17,21,22}\right]$, lo que demuestra que las MV juegan un nuevo papel en esta patología. Sin embargo, el escaso número de MV de células musculares lisas circulantes hace muy difícil su traslación clínica para ser utilizadas como biomarcador que identifique sujetos en riesgo de desarrollar calcificación vascular. Aún así, numerosos estudios sí que han demostrado la utilidad de caracterizar MV endoteliales en plasma como biomarcadores diagnóstico y pronóstico de ECV en diferentes sujetos, incluidos pacientes con ERC $[6,23,24]$. Los resultados de este estudio permiten que se extienda el uso de MV endoteliales en pacientes con ERC que podrían desarrollar CV. Además, el hecho de que las MV endoteliales liberadas experimenten senescencia inducida por IS y promuevan la calcificación en las CMLV podría ofrecer nuevas perspectivas que permitirían el desarrollo de terapias basadas en la modificación de las acciones procalcificadoras de estas MV.

Como se ha comentado, las toxinas urémicas promueven la disfunción endotelial en pacientes con ERC. Esto se ve evidenciado por el hecho de que, entre otras células, las células endoteliales experimentan una senescencia prematura inducida por toxinas urémicas [25-27]. El tratamiento con IS restringe el número de divisiones celulares que ocurren y hacen que las células endoteliales alcancen senescencia prematura inducida por estrés (conocido en inglés como SIPS), que se caracteriza por una detención irreversible en el ciclo celular [28]. SIPS es un mecanismo fisiopatológico de ECV que aparece asociado con ERC [29]. En nuestro modelo in vitro se ha observado un porcentaje creciente de células senescentes en las células endoteliales a lo largo del tratamiento con IS. Esta toxina urémica genera senescencia endotelial prematura en diferentes proporciones, destacando así la susceptibilidad de las células endoteliales, ya que se trata de un cultivo primario y su vida útil es limitada. Los resultados destacan la sensibilidad de las células de cultivo primario de HUVEC tratados con IS.

Además, las células endoteliales dañadas por la toxina urémica IS producen una gran cantidad de MV. Nuestros resultados mostraron que la liberación de MV se incrementó en las células endoteliales tratadas con IS y podría asociarse con el porcentaje de células endoteliales senescentes. En referencia a la liberación de MV de las células endoteliales, algunos estudios mostraron que las células endoteliales dañadas liberan un mayor número de MV [8,9], lo que confirma nuestros datos obtenidos mediante el tratamiento con IS. Estos datos representan una ventaja en la identificación de pacientes con ERC con una lesión endotelial.

Recientemente, nuestro grupo ha demostrado que las células endoteliales que sufren senescencia precoz producen MV que desencadenan el desarrollo de CV [3]. Además, la inducción de senescencia prematura por toxinas urémicas, un mecanismo patológico en las ECV asociadas a la ERC, genera MV que podrían modificar las CMLV. Nuestro enfoque experimental se basa en la exposición de las células vasculares a las MV liberadas por las células endoteliales tratadas con IS, las cuales podrían aumentar la susceptibilidad de CMLV a la transformación ósea y, por lo tanto, promover CV. Nuestros resultados anteriores demostraron que el tratamiento de MV de un modelo de senescencia endotelial replicativa (en inglés conocido como SASP) fue capaz de producir acumulación de calcio en CMLV en el día 9 [3]. Los resultados actuales respaldan el hecho de que las MV de HUVEC dañadas causan un nivel progresivo de acumulación de calcio en las CMLV, que se maximiza en el día 30. Estos datos confirman que las MV endoteliales producen senescencia prematura en pacientes con ERC y causan $\mathrm{CV}$, lo que también se observa durante el envejecimiento fisiológico (modelo de senescencia replicativa in vitro); sin embargo, ambos procesos presentaron diferencias con respecto al desarrollo de CV. Aunque el desarrollo de CV es el paso final en ambos casos, la acumulación de calcio en MV de células endoteliales senescentes se produce de manera diferente porque los tiempos en los que aparecen los depósitos de calcio son diferentes y dependen del tipo de senescencia (SIPS o SASP). Durante el SASP, las MV endoteliales causaron CV por períodos más cortos mientras que, durante el SIPS, las MV liberadas por las células endoteliales dañadas causaron $\mathrm{CV}$ por períodos más largos. Esto implica que las MV específicas presentes antes y durante el proceso de CV deberían ser identificadas para evitar que la patología aparezca en pacientes con ERC. Estos resultados demuestran la existencia de un mecanismo común que subyace al envejecimiento prematuro y la $\mathrm{CV}$ asociada a la edad, además de identificar las diferencias en la línea temporal durante la cual las MV causaron el desarrollo de CV dependiendo del tipo de senescencia inducida por el estímulo (IS) o la fisiología producida por el envejecimiento. Recientemente, Panfoli et al. [30] describieron las MV como herramientas biológicas prometedoras para el diagnóstico y la terapia. Centrarse en las MV específicas que se producen al comienzo del período de desarrollo y durante las últimas etapas de la CV podría ser útil para evitar que la patología aparezca en pacientes con ERC.

\section{Conclusión}

Las células endoteliales se pueden ver dañadas por las toxinas urémicas como el IS presentes en la ERC e inducir una senescencia prematura en las células endoteliales y un aumento de MV que son capaces de promover el desarrollo de CV y, por tanto, las ECV. Nuestro diseño experimental in vitro ha demostrado, 
por primera vez, que las MV representan un nuevo mediador en el mecanismo de transición osteogénica en las células vasculares del músculo liso y, por tanto, en la CV. Estos resultados permitirían el desarrollo de biomarcadores o herramientas terapéuticas en la prevención y tratamiento de pacientes con ECV y/o ERC con riesgo de desarrollar CV mediante la cuantificación en plasma de las MV. Aunque estos datos deben confirmarse mediante la realización de estudios clínicos, los resultados obtenidos respaldan el hecho de que las MV endoteliales en plasma podrían usarse para valorar la progresión de la enfermedad y diseñar nuevas terapias que modulen las alteraciones en la actividad de VE.

\section{Agradecimientos}

Este estudio ha sido subvencionado por el Instituto de Salud Carlos III mediante el proyecto "PI17/01029" y "PI19/00240" (Cofinanciado por el Fondo Europeo de Desarrollo Regional "Una manera de hacer Europa"), Santander/UCM, con el proyecto PR41/17-20964, la Sociedad Española de Nefrologia (proyecto 2019/2020) y los "Proyectos de Investigación del Programa Propio de la UAH", con los proyectos UAH-GP2018-4 y CCG2018/BIO-010.

\section{Bibliografía}

1. Goligorsky MS. Pathogenesis of endothelial cell dysfunction in chronic kidney disease: a retrospective and what the future may hold. Kidney Res Clin Pract. 2015;34(2):76-82. doi:10.1016/j.krcp.2015.05.003

2. Wyatt CM, Drueke TB. Vascular calcification in chronic kidney disease: here to stay? Kidney Int. 2017;92(2):276-278. doi:10.1016/j.kint.2017.05.019

3. Alique M, Ruíz-Torres MP, Bodega G, et al. Microvesicles from the plasma of elderly subjects and from senescent endothelial cells promote vascular calcification. Aging (Albany NY). 2017;9(3):778789. doi:10.18632/aging.101191

4. Raggi P. Cardiovascular disease: Coronary artery calcification predicts risk of CVD in patients with CKD. Nat Rev Nephrol. 2017;13(6):324-326. doi:10.1038/nrneph.2017.61

5. Soriano S, Carmona A, Triviño F, et al. Endothelial damage and vascular calcification in patients with chronic kidney disease. Am J Physiol - Ren Physiol. 2014;307(11):F1302-F1311. doi:10.1152/ajprenal.00114.2014

6. Jansen F, Li Q, Pfeifer A, Werner N. Endothelial- and Immune Cell-Derived Extracellular Vesicles in the Regulation of Cardiovascular Health and Disease. JACC Basic to Transl Sci. 2017;2(6):790807. doi:10.1016/j.jacbts.2017.08.004

7. Yáñez-Mó M, Siljander PRM, Andreu Z, et al. Biological properties of extracellular vesicles and their physiological functions. J Extracell Vesicles. 2015;4(1):27066. doi:10.3402/jev.v4.27066

8. Larson MC, Hillery CA, Hogg N. Circulating membrane-derived microvesicles in redox biology. Free Radic Biol Med. 2014;73:214-228. doi:10.1016/j.freeradbiomed.2014.04.017

9. França CN, de Oliveira Izar MC, do Amaral JB, Tegani DM, Fonseca FAH. Micropartículas como possíveis biomarcadores da doença cardiovascular. Arq Bras Cardiol. 2014;104(2):169-174. doi:10.5935/abc. 20140210

10. Bodega G, Alique M, Puebla L, Carracedo J, Ramírez RM. Microvesicles: ROS scavengers and ROS producers. J Extracell Vesicles. 2019;8(1):1626654. doi:10.1080/20013078.2019.1626654

11. Liu M-L, Williams KJ. Microvesicles: potential markers and mediators of endothelial dysfunction. Curr Opin Endocrinol Diabetes Obes. 2012;19(2):121-127. doi:10.1097/MED.0b013e32835057e9

12. McVey MJ, Kuebler WM. Extracellular vesicles: Biomarkers and regulators of vascular function during extracorporeal circulation. Oncotarget. 2018;9(98):37229-37251. doi:10.18632/oncotarget.26433

13. Carracedo J, Alique M, Ramírez-Carracedo R, Bodega G, Ramírez R. Endothelial Extracellular Vesicles Produced by Senescent Cells: Pathophysiological Role in the Cardiovascular Disease Associated with all Types of Diabetes Mellitus. Curr Vasc Pharmacol. 2018;17(5):447-454. doi:10.2174/1570161116666180820115726

14. Alique M, Ramírez-Carracedo R, Bodega G, Carracedo J, Ramírez R. Senescent microvesicles: A novel advance in molecular mechanisms of atherosclerotic calcification. Int J Mol Sci. 2018;19(7). doi:10.3390/ijms 19072003

15. Lei Q, Liu T, Gao F, et al. Microvesicles as potential biomarkers for the identification of senescence in human mesenchymal stem cells. Theranostics. 2017;7(10):2673-2689. doi:10.7150/thno.18915 
16. Schurgers LJ, Akbulut AC, Kaczor DM, Halder M, Koenen RR, Kramann R. Initiation and Propagation of Vascular Calcification Is Regulated by a Concert of Platelet- and Smooth Muscle Cell-Derived Extracellular Vesicles. Front Cardiovasc Med. 2018;5. doi:10.3389/fcvm.2018.00036

17. Bakhshian Nik A, Hutcheson JD, Aikawa E. Extracellular Vesicles As Mediators of Cardiovascular Calcification. Front Cardiovasc Med. 2017;4(December). doi:10.3389/fcvm.2017.00078

18. Rober S, Poncelet P, Lacroix R, et al. Standardization of platelet-derived microparticle counting using calibrated beads and a Cytomics FC500 routine flow cytometer: a first step towards multicenter studies? J Thromb Haemost. 2009;7(1):190-197. doi:10.1111/j.1538-7836.2008.03200.x

19. Lötvall J, Hill AF, Hochberg F, et al. Minimal experimental requirements for definition of extracellular vesicles and their functions: a position statement from the International Society for Extracellular Vesicles. J Extracell Vesicles. 2014;3(1):26913. doi:10.3402/jev.v3.26913

20. Alique M, Bodega G, Giannarelli C, Carracedo J, Ramírez R. MicroRNA-126 regulates HypoxiaInducible Factor-1 $\alpha$ which inhibited migration, proliferation, and angiogenesis in replicative endothelial senescence. Sci Rep. 2019;9(1):7381. doi:10.1038/s41598-019-43689-3

21. Dusso A, Colombo MI, Shanahan CM. Not all vascular smooth muscle cell exosomes calcify equally in chronic kidney disease. Kidney Int. 2018;93(2):298-301. doi:10.1016/j.kint.2017.08.036

22. Viegas CSB, Santos L, Macedo AL, et al. Chronic kidney disease circulating calciprotein particles and extracellular vesicles promote vascular calcification: A role for GRP (Gla-Rich Protein). Arterioscler Thromb Vasc Biol. 2018;38(3):575-587. doi:10.1161/ATVBAHA.117.310578

23. Dickhout A, Koenen RR. Extracellular Vesicles as Biomarkers in Cardiovascular Disease; Chances and Risks. Front Cardiovasc Med. 2018;5. doi:10.3389/fcvm.2018.00113

24. Chong SY, Lee CK, Huang C, et al. Extracellular vesicles in cardiovascular diseases: Alternative biomarker sources, therapeutic agents, and drug delivery carriers. Int J Mol Sci. 2019;20(13). doi:10.3390/ijms20133272

25. Wolley MJ, Hutchison CA. Large uremic toxins: an unsolved problem in end-stage kidney disease Nephrol Dial Transplant Off Publ Eur Dial Transpl Assoc - Eur Ren Assoc. 2018;33(suppl_3):iii6iii11. doi:10.1093/ndt/gfy179

26. Huang M, Wei R, Wang Y, Su T, Li P, Chen X. The uremic toxin hippurate promotes endothelial dysfunction via the activation of Drp1-mediated mitochondrial fission. Redox Biol. 2018;16:303313. doi:10.1016/j.redox.2018.03.010

27. Saum K, Campos B, Celdran-Bonafonte D, et al. Uremic advanced glycation end products and protein-bound solutes induce endothelial dysfunction through suppression of Krüppel-like factor 2. $J$ Am Heart Assoc. 2018;7(1). doi:10.1161/JAHA.117.007566

28. Ott C, Jung T, Grune T, Höhn A. SIPS as a model to study age-related changes in proteolysis and aggregate formation. Mech Ageing Dev. 2018;170:72-81. doi:10.1016/j.mad.2017.07.007

29. Dai L, Qureshi AR, Witasp A, Lindholm B, Stenvinkel P. Early Vascular Ageing and Cellular Senescence in Chronic Kidney Disease. Comput Struct Biotechnol J. 2019;17:721-729. doi:10.1016/j.csbj.2019.06.015

30. Panfoli I, Santucci L, Bruschi M, et al. Microvesicles as promising biological tools for diagnosis and therapy. Expert Rev Proteomics. 2018;15(10):801-808. doi:10.1080/14789450.2018.1528149 